\title{
SITUACIÓN DE LAS PERSONAS CON DISCAPACIDAD INTELECTUAL Y SUS FAMILIAS FRENTE A LA CRISIS GENERADA POR COVID-19
}

\section{Situation of People with Intellectual Disabilities and their Families in front of the Crisis Generated by COVID-19}

Juan Antonio González Aguilar Fundación Futuro Singular Córdoba juangonzalez@futurosingularcordoba.org

Recepción: 25 de agosto de 2020 Aceptación: 1 de febrero de 2021

RESUMEN: La situación generada por la crisis sanitaria ha desajustado todo el sistema social y las dinámicas habituales de comportamiento de la ciudadanía. Por supuesto, de igual manera, también ha afectado a las personas con discapacidad intelectual y a los sistemas de apoyos para estas. En este artículo queremos describir cómo las personas con discapacidad intelectual a las que Futuro Singular Córdoba presta apoyos vivieron la situación durante el confinamiento, con qué recursos contaban y cómo las familias valoraron el trabajo realizado desde la fundación. Por otra parte, queremos realizar una observación sobre cómo una cuestión de salud pública como la que hemos vivido puede afectar a los modelos de prestación de apoyos a las personas con discapacidad intelectual. Llegando a ponerse en riesgo los avances logrados gracias a enfoques centrados en la persona y de desarrollo comunitario frente a las propuestas de los modelos sociosanitarios que no se ajustan a la realidad de la mayoría de las personas con discapacidad intelectual. Esto supone un claro retroceso en las prácticas de prestación de apoyos centradas en modelos de ciudadanía e impacto en calidad de vida.

Palabras clave: discapacidad intelectual; COVID-19; familias.

AвSTRACT: The situation generated by the health crisis has disrupted the entire social system and the citizens usual behaviour dynamics. Of course, it has also affected people with intellectual disabilities and the support systems for them. In this article we want to 
describe how people with intellectual disabilities to whom Futuro Singular Córdoba provides support lived the situation during the quarantine, what resources they had and how the families valued the work carried out by the foundation. On the other hand, we want to make an observation about how a public health issue like the one we have experienced can affect the models of providing support to people with intellectual disabilities. The progress achieved thanks to person-centered and community development approaches is put at risk in the face of the proposals of social health models that do not adjust to the reality of the majority of people with intellectual disabilities. This represents a clear setback in the practices of providing support focused on models of citizenship and impact on quality of life.

KEYWORDs: intellectual disability; COVID-19; families.

\section{Introducción}

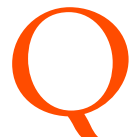

UIZÁs NO HAY GOLPE más fuerte que el más inesperado, y algo así es lo que ha ocurrido. No esperábamos un golpe ni tan duro ni tan demoledor como el que hemos sufrido con la COVID-19. En primer lugar, por la cantidad de personăs que han perdido la vida debido al virus $y$, en segundo lugar, porque ha roto la estructura y dinámica social en la que estábamos habituados a desenvolvernos. Incluso yendo un poco más allá, ha asestado un fuerte golpe a aspectos clave de nuestra cultura. Seguramente nos lleve bastante tiempo digerir esta situación. No obstante, también tenemos una gran oportunidad por delante para aprender y dar respuestas a posibles situaciones futuras, que, aunque no sea idénticas a estas, sí puedan poner en jaque nuestras dinámicas habituales.

En el caso de las personas con discapacidad intelectual y sus familias la realidad ha sido, si es posible, más dura, ya que todos los sistemas de apoyo prácticamente desaparecieron de un día para otro. Las organizaciones que prestan servicios a personas con discapacidad intelectual han tenido que reinventar de manera urgente las dinámicas de apoyo para las personas y las familias en un contexto complejo.

Este artículo pretende mostrar la realidad de las personas con discapacidad intelectual y el impacto que la situación estaba teniendo en sus vidas en pleno confinamiento, basándonos en un estudio realizado en nuestra fundación durante las primeras semanas de la crisis sanitaria.

No obstante, y antes de avanzar los datos de nuestro estudio, es interesante hacer una breve reflexión sobre los modelos de prestación de apoyos, ya que durante este tiempo se ha producido cierto giro hacia modelos sociosanitarios en detrimento de modelos sociales y centrados en calidad de vida.

\subsection{Modelos de prestación de apoyos}

Con la revisión de la definición del concepto de discapacidad intelectual (retraso mental en aquel momento) en 1992 por parte de AAMR (Asociación Americana sobre Retraso Mental, actualmente AAIDD) se inicia un camino que cambiará para 
siempre la orientación de los modelos de intervención con las personas con discapacidad intelectual. Este camino empieza con la propuesta de lo que entendemos como el paradigma de apoyos y que, posteriormente, se desarrolla con los modelos de planificación centrada en la persona y la incorporación del concepto de autodeterminación. Los avances aportados por estos modelos orientan a cambios profundos en el modelo de intervención, evolucionando de un modelo rehabilitador, en el que la persona es parte pasiva, a un modelo que dota de poder a la persona con discapacidad intelectual para que participe activamente en su proyecto de vida, partiendo de las fortalezas de esta y realizando planes de apoyos con base en las necesidades detectadas. Posteriormente, se incorpora a este enfoque el modelo de Calidad de Vida (Schalock y Verdugo, 2002), que permite orientar la prestación de apoyos hacia la obtención de resultados en términos de mejora de la calidad de vida en las personas.

A lo largo de los últimos 20-25 años las organizaciones que prestan apoyos y servicios a las personas con discapacidad intelectual han orientado sus prácticas desde esta perspectiva, alejándose de modelos rehabilitadores y de cuidados básicos para apostar por modelos de apoyos que impulsan el crecimiento y desarrollo de las personas con discapacidad intelectual.

A finales de los 90, e inicio de este nuevo siglo, otro modelo bastante alejado de estos principios entra en escena, el conocido como modelo de atención sociosanitaria. Este se puede definir "como aquel conjunto integrado de servicios y/o prestaciones sociales y sanitarias, públicas y privadas, que se destinan a la rehabilitación y cuidados de personas que se encuentran en diferentes situaciones de dependencia que les impiden realizar actividades personales y/o instrumentales de la vida diaria” (Rodríguez y Monserrat, 2002). Este enfoque se centra de manera clara en los cuidados de salud para personas mayores; de hecho, se plantea como una evolución del modelo sanitario (Fundación Economía y Salud, 2013). No obstante, esta perspectiva se aleja con claridad de los enfoques propuestos por el modelo de calidad de vida, autodeterminación y el paradigma de apoyos.

Con la aparición de la Ley de Promoción de Autonomía Personal y Atención a las Personas en Situación de Dependencia (Ley 39/2006 de 14 de diciembre) la situación se agrava, aún más, para el desarrollo de enfoques centrados en las personas y su promoción. Esta ley, a pesar de su denominación, desde un principio dejó de lado el concepto de "promoción para la autonomía personal” y se centró en el concepto de "dependencia”; de hecho, es conocida como "Ley de dependencia”. Esta orientación hace que, desde un principio, el enfoque de evaluación y prestación de apoyos se haga desde una perspectiva de cuidados al "dependiente" y no de la promoción y desarrollo de las posibilidades de las personas. Un ejemplo de ello es el uso de elementos de evaluación que se centran, casi en exclusiva, en actividades de la vida diaria, obviando otras áreas de conducta adaptativa y desarrollo emocional que son fundamentales para las personas. $\mathrm{O}$ con un enfoque de prestación de recursos y apoyos que apuesta por el modelo de centros alejándose de la perspectiva comunitaria.

Por otra parte, no podemos olvidar que, en relación con los modelos muy centrados en el "cuidado", nace un espacio de negocio muy rentable que, en ocasiones, puede suponer un exceso de presión en la prestación de servicios. 
Estamos observando, con cierta preocupación, que las administraciones se están aproximando más al modelo sociosanitario para la prestación de apoyos a las personas con discapacidad intelectual, lo que supone un retroceso en lo avanzado durante décadas.

\section{Objetivo}

El estudio desarrollado por la Fundación Futuro Singular Córdoba tiene un doble objetivo: por una parte, conocer la realidad por la que las personas con discapacidad intelectual y sus familias estaban pasando durante el confinamiento, conociendo las condiciones de vivienda, la situación del hogar y la vivencia de las personas. Y, por otra parte, saber cómo se estaban percibiendo por parte de las familias los apoyos que en ese momento se estaban prestando desde la fundación.

\section{Método}

\subsection{Participantes}

El estudio se realiza entre los días 15 y 25 de abril, aproximadamente un mes después de la declaración del estado de alarma en España y en un momento de alto impacto del virus. Participan 580 familiares de personas con discapacidad intelectual a las que se les prestan apoyos en servicios no residenciales de la Fundación Futuro Singular Córdoba: atención temprana (22,24\%), colegios $(26,55 \%)$, centros de día $(11,38 \%)$, centros de día ocupacionales $(32,59 \%)$ y centros de recursos $(5,34 \%)$ (un $2 \%$ no indicaron el servicio al que asistía su familiar).

En cuanto a su distribución por localidades el 7,54 \% vivían en poblaciones de menos de 1.500 habs., el $14,15 \%$ en poblaciones de entre 1.500 y 5.000 habs., el $32,53 \%$ en localidades de entre 5.000 y 20.000 habs., el $9,01 \%$ en mayores de 20.000 habs. y el $36,76 \%$ en poblaciones de más de 50.000 habs.

\subsection{Instrumento}

Para el estudio usamos un cuestionario de construcción propia que nos ha permitido indagar en las condiciones en las que las familias afrontaron la situación y si hubo cambios relevantes en algunas áreas. El cuestionario permite valorar cuatro grandes áreas:

- Vivienda: se ofrecía información sobre las características de la vivienda, número de personas que vivían juntas y condiciones tecnológicas.

- Relaciones en el hogar e impacto económico del confinamiento.

- Situación de las personas con discapacidad intelectual: donde se valoraban principalmente la adaptación a la situación y la aparición de conductas disruptivas.

- Valoración de las actuaciones que estaba poniendo en marcha la fundación para seguir prestando apoyos a las personas y familias. 


\subsection{Procedimiento}

Los cuestionarios fueron administrados por los profesionales que habitualmente mantenían contacto con las familias para realizar seguimientos diarios, lo que permitió una mayor cercanía y naturalidad a la hora de abordar los diferentes ítems, especialmente los que arrojaban información de carácter más privado. La disposición de las familias fue plena a participar en el estudio y esto se tradujo en una alta participación.

Por otra parte, queremos destacar las fechas en las que se produce la toma de datos, ya que es uno de los momentos más complicados en relación con el impacto que la pandemia estaba teniendo en el país y en el que la preocupación era máxima, por lo que los datos de este estudio reflejan la situación de ese momento y nos aproximan mucho a la realidad que vivieron las familias y las personas con discapacidad durante el periodo de confinamiento.

\section{Resultados}

Los resultados obtenidos tras la administración del cuestionario serán presentados atendiendo a cuatro grandes bloques: Vivienda, Situación del hogar, Situación de las personas con discapacidad intelectual y Valoración de las acciones desarrolladas por Futuro Singular Córdoba en aquel momento.

\subsection{Vivienda}

En este apartado presentamos los resultados del cuestionario en relación con las condiciones de habitabilidad en que las personas pasaron el confinamiento. En cuanto a los aspectos relacionados con la vivienda se presta atención a variables como el tipo de vivienda, metros cuadrados, si contaban o no con espacio exterior... con la idea de conocer las posibilidades o limitaciones que pudiesen darse por estos motivos. Por otra parte, también se informa sobre los recursos tecnológicos, ya que, al ser la teleasistencia una de las posibilidades de intervención durante el confinamiento, nos era muy interesante saber los medios de los que se disponían.

\subsubsection{Tipo de vivienda}

El tipo de vivienda en que vive la mayoría de las personas son pisos, seguido de casas independientes dentro de la población, siendo la minoría los que viven en el campo. 


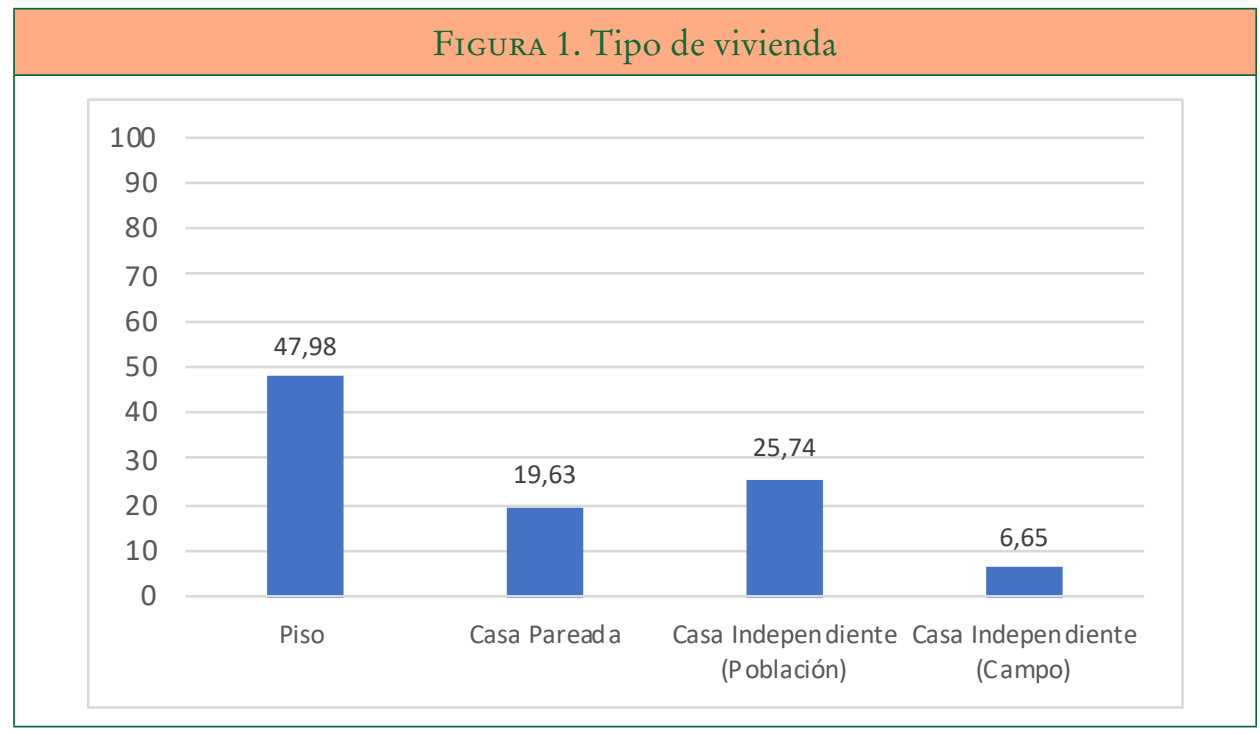

Casi el $59 \%$ contaba con un espacio exterior, como jardín, terraza o patio, siendo el tamaño habitual de las viviendas por debajo de los 120 metros cuadrados.

\begin{tabular}{|l|c|}
\hline \multicolumn{2}{|c|}{ TABLA 1. Distribución de las viviendas por su tamaño } \\
\hline Menos de $90 \mathrm{~m}^{2}$ & $27,68 \%$ \\
\hline Entre $90 \mathrm{~m} 2$ y $120 \mathrm{~m}^{2}$ & $38,88 \%$ \\
\hline Entre $120 \mathrm{~m} 2$ y $200 \mathrm{~m}^{2}$ & $21,36 \%$ \\
\hline Más de $200 \mathrm{~m}^{2}$ & $12,08 \%$ \\
\hline
\end{tabular}

\subsubsection{Medios tecnológicos}

En cuanto a la situación de los medios tecnológicos necesarios para el teletrabajo podemos ver que más del $22 \%$ de las familias no cuentan con internet en sus casas y el $30 \%$ no cuentan con los dispositivos adecuados como ordenadores o tablet. 


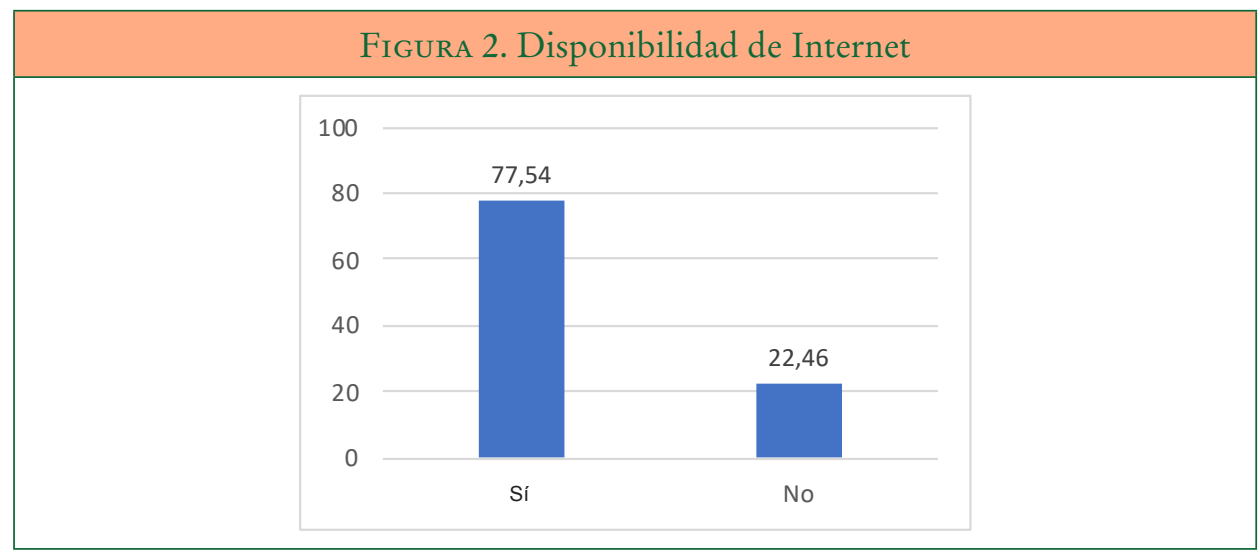

Figura 3. Disponibilidad de dispositivos

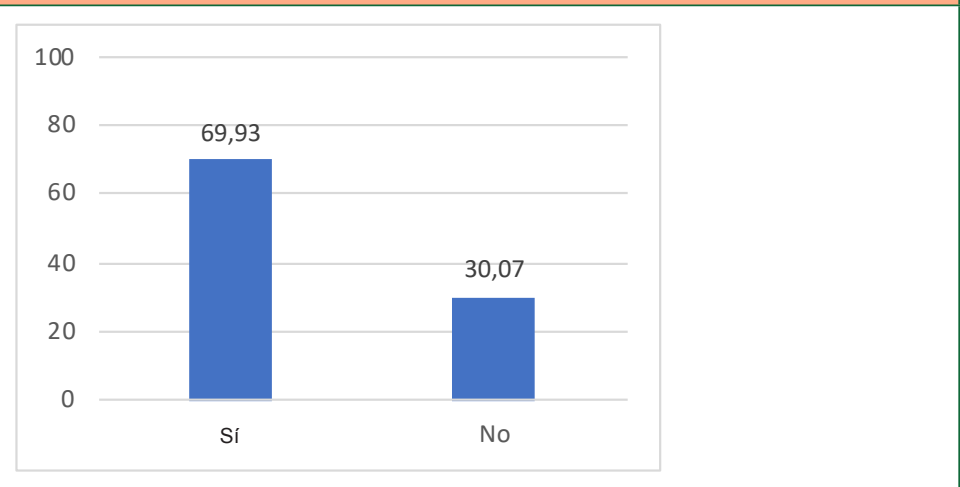

\subsection{Situación en el hogar}

Los datos relacionados con la situación en el hogar muestran la composición de las familias atendiendo al número de personas que viven en el hogar, las relaciones durante la situación de confinamiento y si había más de una persona con necesidades de apoyos en la vivienda.

\subsubsection{Personas en el hogar}

Se observa que en la mayoría de los casos conviven entre 3 y 4 personas, aunque hay situaciones en las que conviven más de seis personas. Y en más del $22 \%$ de los casos conviven más de una persona con necesidades de apoyo. 


\begin{tabular}{|l|l|}
\hline \multicolumn{2}{|c|}{$\begin{array}{c}\text { Tabla 2. Distribución de las familias } \\
\text { por su número de componentes }\end{array}$} \\
\hline 2 personas & $11,29 \%$ \\
\hline 3 personas & $34,40 \%$ \\
\hline 4 personas & $34,91 \%$ \\
\hline 5 personas & $13,58 \%$ \\
\hline 6 personas & $3,70 \%$ \\
\hline 7 personas & $1,06 \%$ \\
\hline 8 personas o más & $1,06 \%$ \\
\hline
\end{tabular}

\begin{tabular}{|l|c|}
\hline \multicolumn{3}{|c|}{ TaBLa 3. Distribución de las familias atendiendo } \\
a si conviven más de una persona con necesidades de apoyo \\
\hline Sí & $22,43 \%$ \\
\hline No & $77,57 \%$ \\
\hline
\end{tabular}

4.2.2. Relaciones en el hogar

En cuanto a las relaciones en el hogar la mayoría de las familias manifiestan que durante este periodo las relaciones han sido buenas $(55,34 \%)$ o muy buenas $(32,74 \%)$.

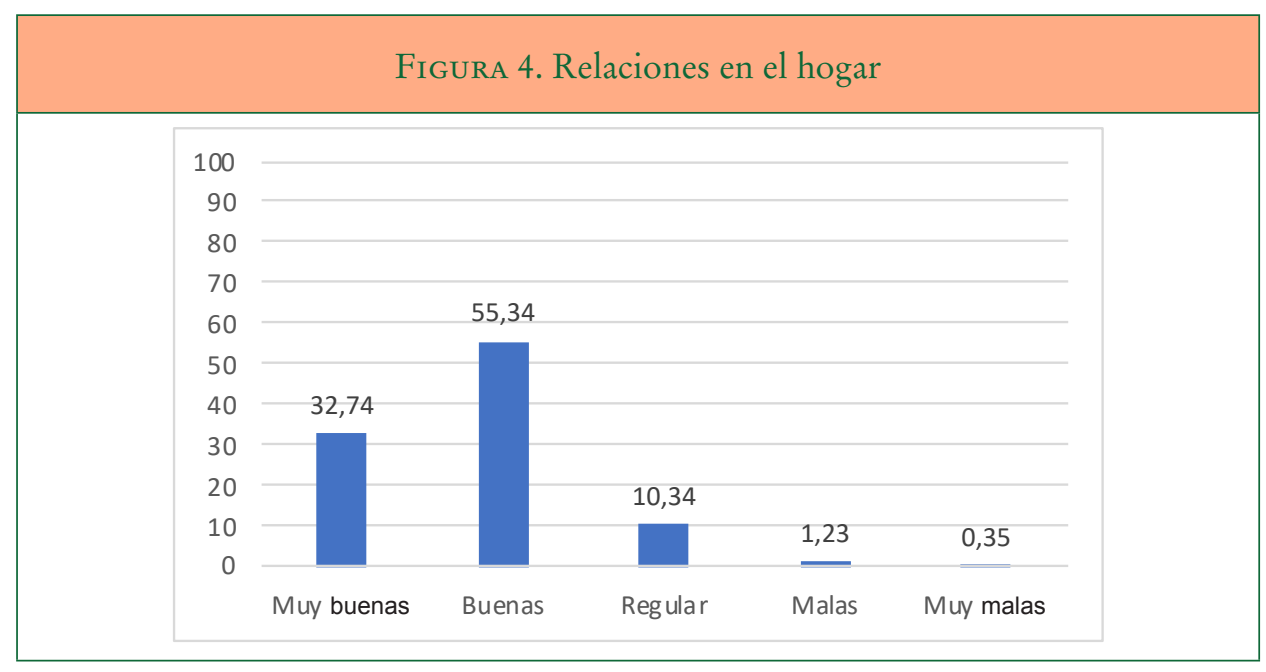

Ediciones Universidad de Salamanca / CC BY-NC-ND

Siglo Cero, vol. 52, número extraordinario, 2021, pp. 183-196 


\subsubsection{Impacto económico}

Esta crisis además de sanitaria está teniendo un alto impacto económico. Cerca del $40 \%$ de las familias han visto afectados sus ingresos habituales, de las cuales, un $10 \%$ han visto disminuidos los ingresos en más del $50 \%$ y más del $5 \%$ en el $100 \%$ de sus ingresos habituales.

\begin{tabular}{|l|l|}
\hline \multicolumn{2}{|c|}{ Tabla 4. Disminución de ingresos por el efecto del confinamiento } \\
\hline No se han visto afectados & $62,86 \%$ \\
\hline Disminución del 25\% & $9,46 \%$ \\
\hline Disminución entre el 25\% y el 50\% & $11,57 \%$ \\
\hline Más del 50\% & $10,51 \%$ \\
\hline Pérdida del $100 \%$ & $5,60 \%$ \\
\hline
\end{tabular}

\subsection{Situación de las personas con discapacidad}

Uno de los aspectos relevantes de este estudio era conocer cómo las personas con discapacidad intelectual se habían adaptado a la situación y si se habían presentado problemas de conducta o conductas disruptivas que, con anterioridad a la situación de confinamiento, no existían. Los resultados los podemos ver de manera general y diferenciados por servicios.

\subsubsection{Adaptación}

Según refieren las familias la adaptación en la mayoría de los casos ha sido buena $(52,09 \%)$ o muy buena $(26,74 \%)$. Siendo mala o muy mala por debajo del $8 \%$.

\begin{tabular}{|l|l|}
\hline \multicolumn{2}{|c|}{$\begin{array}{c}\text { Tabla 5. Adaptación de las personas con discapacidad } \\
\text { a la situación de confinamiento }\end{array}$} \\
\hline Muy bien & $26,75 \%$ \\
\hline Bien & $52,09 \%$ \\
\hline Regular & $13,64 \%$ \\
\hline Mal & $5,95 \%$ \\
\hline Muy mal & $1,57 \%$ \\
\hline
\end{tabular}

Esta situación general cambia en relación con las personas con más necesidades de apoyos, como reflejan los datos de centro de día, en el que la adaptación ha sido peor. 
TaBla 6. Adaptación de las personas con discapacidad a la situación de confinamiento en función de los servicios

\begin{tabular}{|l|c|c|c|c|c|}
\hline & Muy bien & Bien & Regular & Mal & Muy Mal \\
\hline Atención Temprana & $34,11 \%$ & $51,16 \%$ & $7,75 \%$ & $6,20 \%$ & $0,78 \%$ \\
\hline Colegios & $17,45 \%$ & $58,39 \%$ & $16,11 \%$ & $6,71 \%$ & $1,34 \%$ \\
\hline Centros de día & $4,55 \%$ & $43,94 \%$ & $28,78 \%$ & $16,67 \%$ & $6,06 \%$ \\
\hline Centros ocupacionales & $38,30 \%$ & $48,93 \%$ & $10,11 \%$ & $1,60 \%$ & $1,06 \%$ \\
\hline Centro de recursos & $9,68 \%$ & $74,19 \%$ & $16,63 \%$ & - & - \\
\hline
\end{tabular}

\subsubsection{Aparición de conductas disruptivas}

Un aspecto importante a indagar fue la posible aparición de conductas desajustadas debido a la situación de confinamiento. Según los datos obtenidos estas han aparecido en el 19,47\% de los casos, siendo significativamente más alta $(28,79 \%)$ en las personas con más necesidades de apoyo, como se desprende de los datos de los servicios de día. Igualmente ha ocurrido en los casos de Atención Temprana $(32 \%)$.

\begin{tabular}{|l|l|l|}
\hline \multicolumn{3}{|c|}{ Tabla 7. Aparición de conductas desajustadas en función de los servicios } \\
\hline & Sí & No \\
\hline Atención Temprana & $32,03 \%$ & $66,97 \%$ \\
\hline Colegios & $20,95 \%$ & $79,05 \%$ \\
\hline Centros de día & $28,79 \%$ & $71,21 \%$ \\
\hline Centros ocupacionales & $6,92 \%$ & $93,08 \%$ \\
\hline Centro de recursos & $12,90 \%$ & $87,10 \%$ \\
\hline Global servicios & $19,47 \%$ & $80,53 \%$ \\
\hline
\end{tabular}

\subsection{Valoración de las acciones realizadas por la fundación}

Desde el primer día posterior al decreto del estado de alarma desde la fundación se pusieron en marcha diferentes medidas con la intención de acompañar a las familias y prestar los apoyos posibles dadas las circunstancias. En este sentido, era importante saber si las medidas adoptadas estaban aportando valor a las familias en una situación tan complicada. Se valoran tres aspectos: el seguimiento realizado por parte de los profesionales, las actividades on-line desarrolladas y la satisfacción con la atención recibida. 


\subsubsection{Seguimiento}

Una de las principales medidas fue realizar acciones de acompañamiento y seguimiento a las familias. Estas acciones han sido valoradas muy positivamente por las familias, ya que el $80 \%$ de ellas lo han valorado entre 9 y 10 .

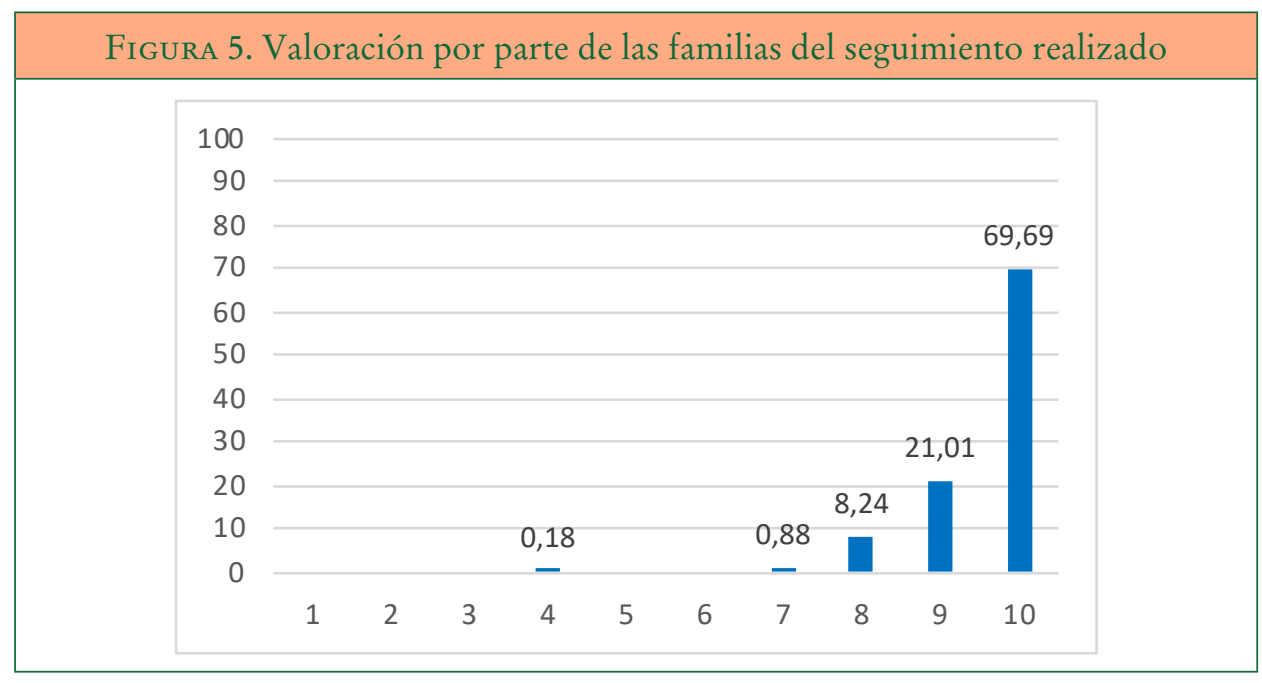

\subsubsection{Actividades on-line}

Por otra parte, se incorporaron estrategias de apoyo a las personas con discapacidad intelectual basadas en metodología on-line (videoconferencias, teléfono, etc.). Igualmente, estas medidas han sido valoradas positivamente por las familias: el $95 \%$ las valoró entre 8 y 10. 
Figura 6. Valoración por parte de las familias de las actividades on-line propuestas

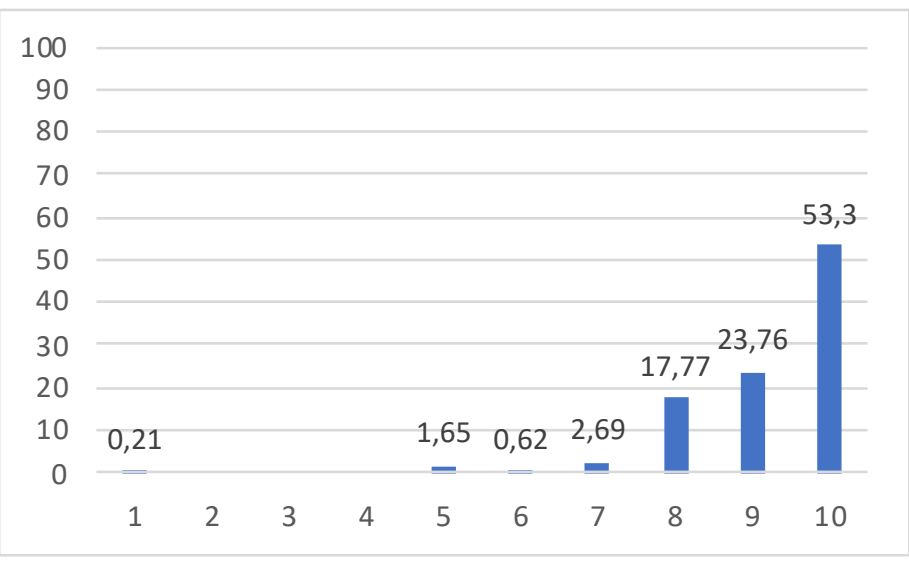

\subsubsection{Satisfacción con la atención}

Finalmente, nos interesamos por la valoración que las familias han realizado sobre la atención recibida desde la fundación y el centro en los momentos de confinamiento. Más del $90 \%$ de las familias han valorado estas actuaciones con una puntuación de entre 9 y 10.

FIGURA 7. Satisfacción por parte de las familias de la atención recibida

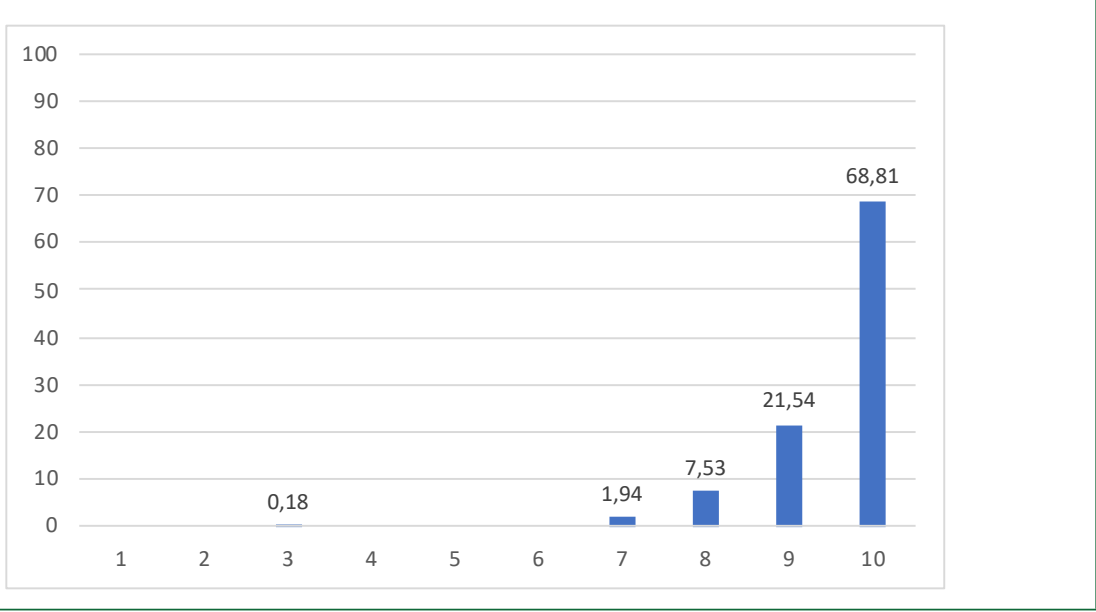

Ediciones Universidad de Salamanca / CC BY-NC-ND

Siglo Cero, vol. 52, número extraordinario, 2021, pp. 183-196 


\section{Discusión}

El objetivo de este trabajo era conocer y mostrar la realidad de las personas con discapacidad intelectual y sus familias en los primeros momentos del confinamiento, así como conocer el impacto que la situación estaba teniendo en sus vidas.

En relación con la capacidad, medios y conocimientos, para prestar los apoyos necesarios a las personas y familias de manera telemática, nos hemos encontrado que nuestro tejido social no está tan preparado como sería necesario para ofrecer soluciones basadas en la tecnología. Hay carencias importantes en aspectos tan básicos como el acceso a internet o disponer de los dispositivos adecuados.

Respecto al impacto de la situación de confinamiento en las relaciones dentro del marco familiar hemos constatado que las familias han soportado perfectamente esta prueba de tensión. No obstante, han surgido situaciones críticas, como el aumento de conductas desajustadas, especialmente en el caso de personas con más necesidades de apoyo. De nuevo podemos constatar que las personas con altas necesidades de apoyos necesitan dispositivos más ajustados, no siendo válidas las mismas propuestas que para personas con menos necesidades de apoyos.

Por otra parte, las personas con discapacidad intelectual han demostrado una gran capacidad de adaptación en estas circunstancias tan adversas. Esto pone, de nuevo, de manifiesto que el concepto de discapacidad está más en relación con las exigencias del entorno y los apoyos prestados que con las capacidades y limitaciones de las personas.

En cuanto al impacto económico de esta crisis, está siendo el más importante que hemos sufrido en muchos años. Gran número de las familias que han participado en el estudio han visto afectado su nivel de ingresos de manera significativa. La recuperación económica no será rápida, lo que puede agravar la situación de muchas familias que ya padecían el sobrecoste para mantener los apoyos que las personas necesitan.

\section{Conclusiones}

La situación generada por las medidas de confinamiento supuso un importante reajuste en la dinámica habitual de las familias y las personas con discapacidad intelectual. Las medidas, que fueron adoptadas para la población en general, tardaron en adaptarse a las necesidades de las personas con discapacidad intelectual, si es que se adaptaron. Esto ha llevado, en muchos casos, a tener que actuar con cierta improvisación ya que no se tenían en cuenta las variables que afectaban a las personas con discapacidad y a sus familias. Claros ejemplos de ello son las necesidades de medios tecnológicos que las familias tenían para la prestación de servicios o apoyos. O las limitaciones de movimiento que se impusieron sin tener en cuenta que muchas personas, debido a sus necesidades cognitivas y conductuales, no podían permanecer en sus casas todas las horas del día, lo que en muchos casos generó dificultades conductuales. 
Como indicábamos al principio, nos hemos tenido que enfrentar a una situación que, tanto por inesperada como por la gravedad de su impacto en la vida de las personas, ha puesto en crisis nuestro sistema social y, por supuesto, el sistema de prestación de apoyos a las personas con discapacidad intelectual. No obstante, también hemos podido experimentar que, aun en un escenario tan adverso, hay oportunidades para hacer las cosas de otra manera y de esta forma abrir nuevas vías de prestación de apoyos, tanto para las personas con discapacidad intelectual como para sus familias.

Finalmente, me gustaría incidir en una de las ideas iniciales y es que tenemos que velar por el mantenimiento y desarrollo de modelos comunitarios y de ciudadanía frente a modelos sociosanitarios que tienden a la medicalización de los servicios y la vuelta a paradigmas anteriores. Es por ello por lo que el reto es doble: por una parte, superar la situación de crisis actual sin dejar a un lado a las personas con discapacidad intelectual, adaptando la prestación de apoyos a nuevos contextos. Y, por otra parte, hacerlo desde el impulso de modelos inclusivos y que apuestan por el desarrollo comunitario de las personas con discapacidad intelectual.

\section{Referencias bibliográficas}

Fundación Economía y SALud. (2013). Evolucionando hacia un modelo sociosanitario de salud. F. Economía y Salud.

Luckasson, R., Borthwick-Duffy, S., Buntinx, W. H., Coulter, D. L., Craig, E. M. P., Reeve, A., Schalock, R. L., Snell, M. E., Spitalnik, D. M., Spreat, S. y Tassé, M. J. (2002). Mental retardation: definition, classification, and systems of supports. American Association on Mental Retardation.

Luckasson, R., Coulter, D. L., Polloway, E. A., Reiss, S., Schalock, R. S., Shell, M. E.,... y STARK, J. A. (1992). Mental retardation: definition, classification, and systems of support. American Association on Mental Retardation.

Rodríguez, G. y Monserrat, J. (2002). Modelos de atención sociosanitaria. Una aproximación a los costes de la dependencia. IMSERSO, Ministerio de Trabajo y Asuntos Sociales.

Schalok, R. L. y Verdugo, M. Á. (2003). Calidad de vida: manual para profesionales de la educación, salud y servicios sociales. Alianza. 